\title{
Radiative Width of the Hoyle State from $\gamma$-Ray Spectroscopy
}

\author{
T. Kibédi®, ${ }^{1, *}$ B. Alshahrani®,$^{1,2, \dagger}$ A. E. Stuchbery $\odot,{ }^{1}$ A. C. Larsen $\odot,{ }^{3}$ A. Görgen $\odot,{ }^{3}$ S. Siem $\odot,{ }^{3}$ M. Guttormsen $\odot,{ }^{3}$ \\ F. Giacoppo, ${ }^{3,3}$ A. I. Morales $\odot,{ }^{4,8}$ E. Sahin, ${ }^{3}$ G. M. Tveten, ${ }^{3}$ F. L. Bello Garrote, ${ }^{3}$ L. Crespo Campo, ${ }^{3}$ \\ T. K. Eriksen $\odot,{ }^{3}$ M. Klintefjord, ${ }^{3}$ S. Maharramova $\odot,{ }^{3}$ H.-T. Nyhus, ${ }^{3}$ T. G. Tornyi ${ }^{3,5, \|}$ \\ T. Renstrøm, ${ }^{3}$ and W. Paulsen ${ }^{3}$ \\ ${ }^{1}$ Department of Nuclear Physics, Research School of Physics, The Australian National University, \\ Canberra, Australian Capital Territory 2601, Australia \\ ${ }^{2}$ Department of Physics, Faculty of Science, King Khalid University, Abha 61413, Saudi Arabia \\ ${ }^{3}$ Department of Physics, University of Oslo, N-0316 Oslo, Norway \\ ${ }^{4}$ Dipartimento di Fisica dell'Universitá degli Studi di Milano and INFN-Milano, 20133 Milano, Italy \\ ${ }^{5}$ Institute of Nuclear Research, MTA ATOMKI, Debrecen H-4026, Hungary
}

(Received 18 December 2019; revised 26 July 2020; accepted 22 September 2020; published 27 October 2020)

\begin{abstract}
The cascading 3.21 and $4.44 \mathrm{MeV}$ electric quadrupole transitions have been observed from the Hoyle state at $7.65 \mathrm{MeV}$ excitation energy in ${ }^{12} \mathrm{C}$, excited by the ${ }^{12} \mathrm{C}\left(p, p^{\prime}\right)$ reaction at $10.7 \mathrm{MeV}$ proton energy. From the proton- $\gamma-\gamma$ triple coincidence data, a value of $\Gamma_{\mathrm{rad}} / \Gamma=6.2(6) \times 10^{-4}$ was obtained for the radiative branching ratio. Using our results, together with $\Gamma_{\pi}^{E 0} / \Gamma$ from Eriksen et al. [Phys. Rev. C 102, $024320(2020)]$ and the currently adopted $\Gamma_{\pi}(E 0)$ values, the radiative width of the Hoyle state is determined as $\Gamma_{\text {rad }}=5.1(6) \times 10^{-3} \mathrm{eV}$. This value is about $34 \%$ higher than the currently adopted value and will impact models of stellar evolution and nucleosynthesis.
\end{abstract}

DOI: 10.1103/PhysRevLett.125.182701

The triple-alpha reaction, which produces stable ${ }^{12} \mathrm{C}$ in the Universe, is a fundamental process of helium burning stars. The entry state of the triple-alpha process, the second excited state in ${ }^{12} \mathrm{C}$, is a $0^{+}$state at $7.65 \mathrm{MeV}$. It has attracted significant attention [1-3] since it was first proposed in 1953 by Hoyle [4]. The existence of the state was confirmed in the same year from the analysis of the $\alpha$ spectrum from the ${ }^{14} \mathrm{~N}(d, \alpha){ }^{12} \mathrm{C}$ reaction [5]. The Hoyle state is $\alpha$ unbound and the dominant decay process (>99.94\%) is through the emission of an $\alpha$ particle, leading to the very short-lived isotope ${ }^{8} \mathrm{Be}$, which then disintegrates into two $\alpha$ particles. Stable carbon will only be produced either if the Hoyle state decays directly to the ground state via an electric monopole (E0) transition or by a cascade of two electric quadrupole (E2) transitions.

Because of its unusual structure, the Hoyle state has attracted continuous attention; see the recent review of Freer and Fynbo [1] and other recent works [2,6,7]. The discussion includes nuclear clustering, a spacial arrangement of the three $\alpha$ particle clusters of which the state is believed to be composed, and discussion on a new form of nuclear matter, in analogy with the Bose-Einstein condensates. The characterization of the $2^{+}$and $4^{+}$states on top of the $7.65 \mathrm{MeV}^{+}$state, forming the Hoyle band [8], together with much improved ab initio calculations [9] are important steps forward.

The production rate of stable carbon in the Universe is cardinal for many aspects of nucleosynthesis. The reaction rate is closely related to the decay properties of the Hoyle state. The triple-alpha reaction rate can be expressed as $r_{3 \alpha}=\Gamma_{\text {rad }} \exp \left(-Q_{3 \alpha} / k T\right)$ [10]. Here $\Gamma_{\text {rad }}$ is the total electromagnetic (radiative) decay width, $Q_{3 \alpha}$ is the energy release in the three $\alpha$ breakup of the Hoyle state, and $T$ is the stellar temperature. $\Gamma_{\text {rad }}$ has contributions from the $3.21 \mathrm{MeV} E 2$ and the $7.65 \mathrm{MeV}$ E0 transitions. The contributions of electron conversion are negligible, so including photon $(\gamma)$ and pair conversion $(\pi), \Gamma_{\mathrm{rad}}=$ $\Gamma_{\gamma}^{E 2}+\Gamma_{\pi}^{E 2}+\Gamma_{\pi}^{E 0}$. Based on current knowledge, $98.4 \%$ of the electromagnetic decay width is from the $E 2$ photon emission and $1.5 \%$ is from the $E 0$ pair decay [11]. The $\Gamma_{\pi}^{E 2}$ contribution is less than $0.1 \%$.

The value of $\Gamma_{\text {rad }}$ cannot be directly measured. It is usually evaluated as a product of three independently measured quantities:

$$
\Gamma_{\mathrm{rad}}=\left[\frac{\Gamma_{\mathrm{rad}}}{\Gamma}\right]\left[\frac{\Gamma}{\Gamma_{\pi}(E 0)}\right]\left[\Gamma_{\pi}(E 0)\right],
$$

where $\Gamma$ is the total decay width of the Hoyle state, which includes the $\alpha$ as well as the E2 and E0 electromagnetic decays.

The only absolute quantity in Eq. (1) is $\Gamma_{\pi}(E 0)$, which has been measured 8 times [12-19]. The two most recent measurements $[18,19]$ are the most precise; however, they disagree by more than $5 \sigma$. Following the recommendation of Freer and Fynbo [1], we have adopted a value of $62.3(20) \mu \mathrm{eV}$ from the latter study. 
The least precisely known quantity is $\Gamma_{\pi}(E 0) / \Gamma$. Combining all previous measurements [20-24], a value of $\Gamma_{\pi}(E 0) / \Gamma=6.7(6) \times 10^{-6}$ was adopted [1]. This value has been further improved by a new pair conversion measurement at the Australian National University [25] and a $\Gamma_{\pi}(E 0) / \Gamma$ ratio of $7.6(4) \times 10^{-6}$ was recommended.

The third term, $\Gamma_{\text {rad }} / \Gamma$, has been measured 8 times between 1961 and 1976 [26-33]. By excluding the value of $2.8(3) \times 10^{-4}$ by Seeger and Kavanagh [27], the weighted mean value is $4.13(11) \times 10^{-4}$. In Ref. [1], a slightly higher value of $4.19(11) \times 10^{-4}$ was recommended. $\Gamma_{\text {rad }} / \Gamma$ is claimed to be the most precise term in Eq. (1).

In the present Letter we report a new measurement of $\Gamma_{\gamma}^{E 2} / \Gamma$, which was deduced from the rate of proton $-\gamma-\gamma$ triple coincidences $N_{020}^{7.65}$ corresponding to the deexcitation of the Hoyle state through the emission of the 3.21 and $4.44 \mathrm{MeV} \gamma \gamma$ cascade, to the rate of singles proton events $N_{\text {singles }}^{7.65}$ exciting the Hoyle state:

$$
\frac{\Gamma_{\gamma}^{E 2}}{\Gamma}=\frac{N_{020}^{7.65}}{N_{\text {singles }}^{7.65} \times \epsilon_{3.21} \times \epsilon_{4.44} \times W_{020}^{7.65}},
$$

where $\epsilon_{3.21}$ and $\epsilon_{4.44}$ are the photon detection efficiencies and $W_{020}^{7.65}$ is the angular correlation correction for a $0-2-0$ cascade. Our approach is similar to that of Obst and Braithwaite [33], but with much improved experimental apparatus and analysis techniques.

The experiments were carried out at the Cyclotron Laboratory of the University of Oslo. The Hoyle state was populated in inelastic scattering of $10.7 \mathrm{MeV}$ protons on a $180 \mu \mathrm{g} / \mathrm{cm}^{2}$ natural carbon target. This energy was slightly higher than the notional optimum energy of $10.5 \mathrm{MeV}$, where the ${ }^{12} \mathrm{C}\left(p, p^{\prime}\right)$ reaction has a relatively broad resonance [34]. The higher proton energy was employed to shift the inelastically scattered protons to $\sim 1.5 \mathrm{MeV}$, well above the detecting threshold. Proton angular distribution measurements [25] suggest that the ratio of the excitation of the 4.44 and $7.65 \mathrm{MeV}$ states is essentially the same at 10.5 and $10.7 \mathrm{MeV}$. In the present experiments a beam intensity of $5 \mathrm{nA}$ was used, keeping the total count rate below $3 \mathrm{kHz}$. Additional experiments were carried out using a target consisting of a layer of $140 \mu \mathrm{g} / \mathrm{cm}^{2} \mathrm{SiO}_{2}$ on a $32 \mu \mathrm{g} / \mathrm{cm}^{2}$ natural carbon backing. The ${ }^{28} \mathrm{Si}\left(p, p^{\prime}\right)$ reaction was used to determine the photon detection efficiencies. The $0^{+}$state at $4.98 \mathrm{MeV}$ in ${ }^{28} \mathrm{Si}$ decays with a $100 \%$ branching ratio to the ground state through the emission of a $3.20-1.78 \mathrm{MeV}$ cascade. In addition, the 4.50-1.78 MeV cascade from the $6.28 \mathrm{MeV}$ $3^{+}$state was also analyzed. The branching ratio of this cascade is $B_{\gamma}^{6.28}=88.2(4) \%$ [35].

Proton $-\gamma-\gamma$ coincidences were measured with the SiRi particle telescope [36] and the CACTUS $\gamma$-ray detector array [37]. The $64 \Delta E-E$ telescopes of SiRi were placed in the backward direction covering angles between $126^{\circ}$

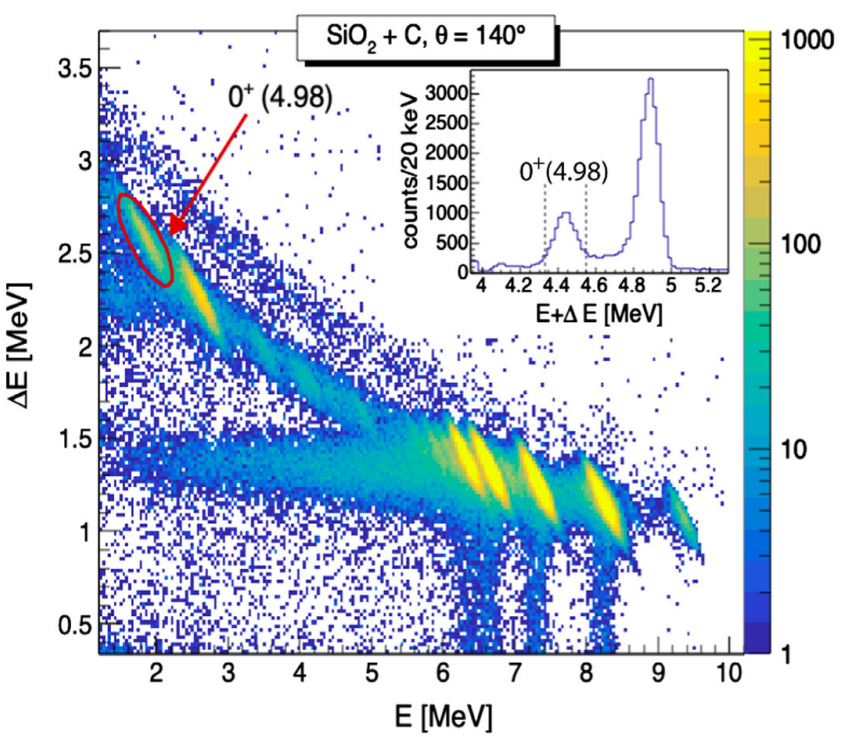

FIG. 1. Singles proton events recorded in the SiRi $E$ (horizontal axis) versus $\Delta E$ (vertical axis) telescopes using the $\mathrm{SiO}_{2}$ plus carbon target. Events corresponding to the excitation of the $4.98 \mathrm{MeV}^{+}$state are indicated by the ellipse. The inset shows $\Delta E+E$ total energy spectrum around the proton group of the $4.98 \mathrm{MeV}^{+}$state, together with the energy gate (dashed lines).

and $140^{\circ}$ relative to the beam direction. The solid angle of the particle detection was around $6 \%$ of $4 \pi$. The front $(\Delta E)$ and back $(E)$ particle detectors have thicknesses of 130 and $1550 \mu \mathrm{m}$, respectively. $\gamma$ rays were recorded with the CACTUS array consisting of 26 collimated $5^{\prime \prime} \times 5^{\prime \prime} \mathrm{NaI}$ (Tl) detectors, placed at $22 \mathrm{~cm}$ from the target. Each detector had a $10 \mathrm{~cm}$ lead collimator to ensure illumination of the center of the detector. The total photon efficiency of the array is $\approx 14.2 \%$ of $4 \pi$ at $1.33 \mathrm{MeV}$ energy.

Signals in the $\Delta E$ detectors were used as triggers and to start the time-to-digital converter (TDC). The stop signal was generated when any $\mathrm{NaI}(\mathrm{Tl})$ detector fired. In this way prompt proton- $\gamma-\gamma$ coincidences could be sorted from the event-by-event data. Figure 1 shows the energy deposition in the $E$ versus $\Delta E$ detectors recorded with the $\mathrm{SiO}_{2}$ plus carbon target. The fraction of the particle energy deposited in the front detector depends on $Z, A$, and the particle energy. This relation, visible in Fig. 1 as a "banana-shaped" region, can be used to identify the detected particles, and also to filter events of incomplete energy deposition (horizontal and vertical bands), as well as other beam related background events. The $\Delta E-E$ spectrum can be used to select the population of specific states. Protons exciting the Hoyle state fully stop in the $\Delta E$ detector. In this case the $\Delta E-E$ telescope was operated in anticoincidence to reject high energy particle events depositing only partial energy in the $\Delta E$ detector.

Figure 2 shows the spectra of singles proton and $\gamma$-ray events from the ${ }^{12} \mathrm{C}\left(p, p^{\prime}\right)$ reaction collected over a period of 12 days. The peak at $1.5 \mathrm{MeV}$ proton energy, labeled as 


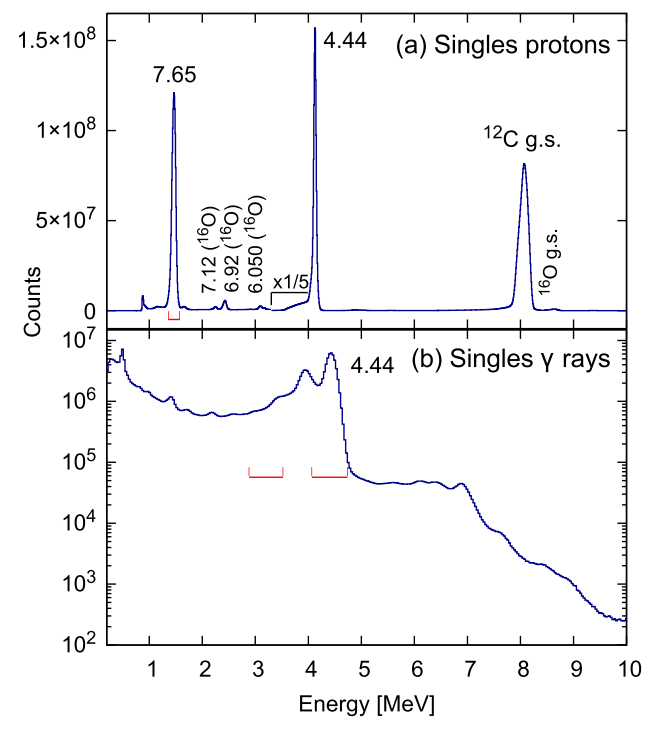

FIG. 2. Singles spectra of (a) protons and (b) $\gamma$ rays using the ${ }^{12} \mathrm{C}\left(p, p^{\prime}\right)$ reaction. The proton $\left(7.65_{p}\right)$ and $\gamma$-ray energy $\left(3.21_{\gamma}\right.$ and $4.44_{\gamma}$ ) gates used for the analysis are indicated by red lines.

" 7.65 ", represents the excitation of the Hoyle state. It contains $N_{\text {singles }}^{7.65}=2.78(6) \times 10^{8}$ events; however, only 1 out of $\sim 2500$ proton excitations is expected to result in electromagnetic transitions leading to the ground state of ${ }^{12} \mathrm{C}$. In comparison, the number of protons exciting the $4.44 \mathrm{MeV}^{+}$state is about 4.7 times higher, and this state always decays to the ground state with an E2 $\gamma$-ray transition. The singles $\gamma$-ray spectrum, shown in Fig. 2(b), is dominated by the $4.44 \mathrm{MeV}$ photon events. Beside the full energy peak, there is a broad distribution of events of single (at $\sim 3.9 \mathrm{MeV}$ ) and double (at $\sim 3.4 \mathrm{MeV}$ ) escapes, as well as Compton scattering. The $3.21 \mathrm{MeV}$ transition is expected to be about 10000 times weaker and it partially overlaps with the second escape peak of the 4.44 MeV line. In this energy region the photon energy resolution was around $0.19 \mathrm{MeV}$. Excitation of the $4.44 \mathrm{MeV}$ state will only produce a single photon event. However, we estimated that the probability of two 4.44 $\mathrm{MeV} \gamma$ rays produced by two unrelated reactions and observed in prompt coincidence is $7 \times 10^{-5}$ per sec, which is about 3 times lower than the true coincidence rate and can be considered as high.

Figure 3 shows the time differences between protons exciting the Hoyle state and a pair of 3.21 and $4.44 \mathrm{MeV}$ $\gamma$ rays. The main peak at $\Delta T(p \gamma)=0 \mathrm{~ns}$ ("pr") corresponds to $\gamma$ rays in prompt coincidence with protons. The secondary peaks ("bgLx" and "bgRx") occurring every $72 \mathrm{~ns}$ are from accidental coincidences where one of the two $\gamma$ rays was produced in another beam burst. The 4 background gates either side of the prompt and equal width to the prompt peak were averaged over.

Figure 4(a) shows protons ("7.65") in prompt coincidence with a 3.21 and a $4.44 \mathrm{MeV} \gamma$ ray without subtraction

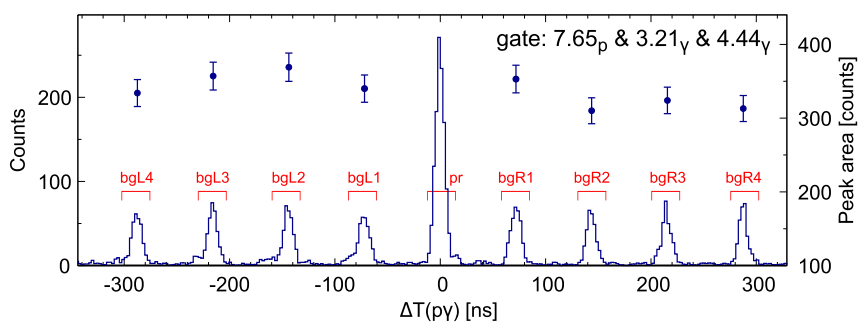

FIG. 3. Time differences between protons exciting the Hoyle state and 3.21 and $4.44 \mathrm{MeV} \gamma$ rays. The prompt (pr) and four background gates on each side (bgLx, bgRx) are marked in red. The average counts in the background peaks is $318(18)$.

of accidental coincidences. In the same spectrum $N_{p}\left(2_{1}^{+}\right)$, the number of protons exciting the $2_{1}^{+}$state ("4.44") is due to accidental coincidences and is nearly 50 times higher. Using TDC gates of "pr-pr", "pr-bgLx", "pr-bgRx", and "bgLx-bgRx", the numbers of $N_{p}\left(2_{1}^{+}\right)$ events in the corresponding proton spectra are 8251(91), 7697(88), 7914(89), and 54(9), respectively. Protons exciting the $2_{1}^{+}$state will only produce single photon events; therefore, the $N_{p}\left(2_{1}^{+}\right)$rates can be used to remove the random events. Using the above $N_{p}\left(2_{1}^{+}\right)$rates the scaling factor was obtained as 8251(91)/ $\{[7697(88)+7914(89)-$ $54(9)] / 2\}=1.061(12)$. The $N_{p}\left(0^{+}\right)$rates in the same TDC gates were 249(16), 158(13), 197(14), and 66(8), respectively. This gives $N_{020}^{7.65}=212(22)$ counts. The final proton spectrum in triple coincidence with the 3.21 and $4.44 \mathrm{MeV} \gamma$ rays is shown in Fig. 4(b).

Figure 5 shows the $\gamma-\gamma$ coincidence events gated by protons exciting the Hoyle state, where the horizontal axis is the $\gamma$-ray energy and the vertical axis is the summed energy of the two $\gamma$ rays in coincidence. The number of random events has been evaluated using the accidental coincidences of the $4.44 \mathrm{MeV} \gamma$ ray with itself, indicated as "4.44/4.44". The number of such events in the various TDC

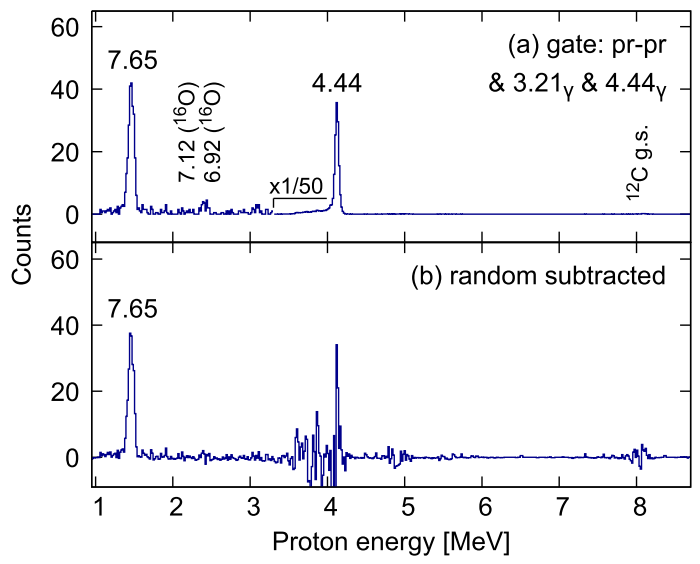

FIG. 4. Protons in prompt coincidence with the 3.21 and $4.44 \mathrm{MeV} \gamma$-rays cascade. (a) Both $\gamma$ rays observed in the prompt ("pr-pr") TDC window, and (b) random events from the "pr-bgLx" and "pr-bgRx" TDC gates (Fig. 3) are subtracted. 


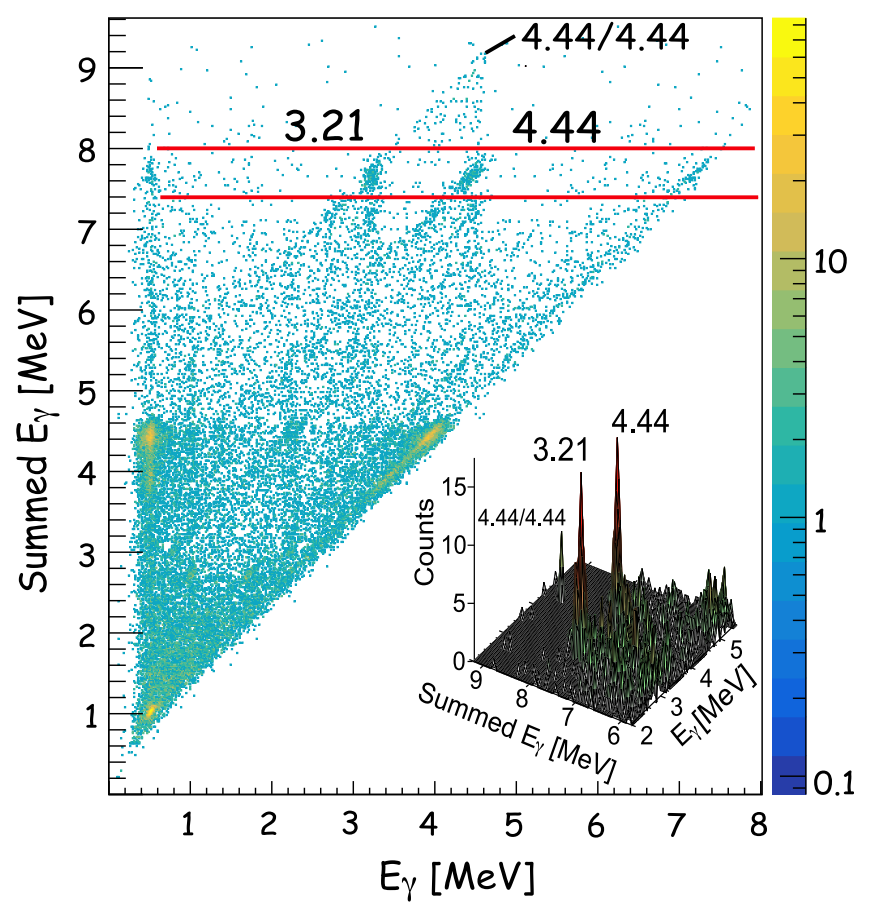

FIG. 5. $\gamma$-ray energy versus summed $\gamma$-ray energy matrix constructed from $\gamma-\gamma$ coincidence events gated by protons exciting the Hoyle state. Random events have been removed. The gate representing the 3.21 plus $4.44 \mathrm{MeV}$ summed energy $\left(7.65_{\text {sum }}\right)$ is indicated with red horizontal lines. The inset shows the region around the 3.21 and $4.44 \mathrm{MeV}$ transitions in 3D. Data have been compressed by factor 4 . The location of the random coincidences of the $4.44 \mathrm{MeV} \gamma$ ray with itself is also marked.

gates were 131(12), 157(13), 134(12), 63(8), which gives a subtraction factor of 1.15(11), a value consistent with the one obtained from the proton spectra. To deduce the final $\gamma \gamma$ coincidence spectra, the scaling factor of 1.061(12) was adopted. Figure 5 also shows the final matrix of $\gamma \gamma$ coincidence events. A small residue of the 4.44-4.44 random coincidences is visible, but the number of related events under the peaks of interest is negligible.

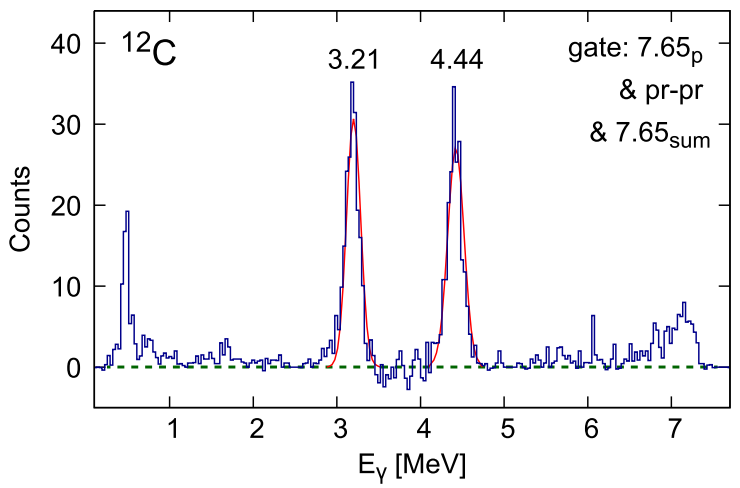

FIG. 6. Random subtracted $\gamma$ rays from the Hoyle state. The fit to the spectrum including the 3.21 and $4.44 \mathrm{MeV}$ transitions is shown in red.
The final $\gamma$-ray spectrum of the $3.21-4.44 \mathrm{MeV}$ cascade is shown in Fig. 6. The areas of the 3.21 and $4.44 \mathrm{MeV}$ photon peaks, 208(21) and 213(21) counts, were obtained by fitting Gaussian functions to these data.

Using the scaling factor of 1.061(12), the true triple coincidence events in the prompt $p \gamma$ peak in Fig. 3 were evaluated as $N_{020}^{7.65}=237(23)$. The adopted value of the $N_{020}^{7.65}=217(21)$ was obtained as the weighted mean of the three values deduced from the different projections.

The absolute photon detection efficiency $\epsilon$ was evaluated using the PENELOPE code [38]. The same simulations were used to evaluate the correction factors, $W_{020}$ and $W_{320}$, for the $\gamma$-ray angular correlation, including geometrical attenuation coefficients [39], listed in Table I. To confirm the accuracy of the simulations, the proton gated spectrum of the 1.78 and $4.50 \mathrm{MeV} \gamma$ rays from the $6.28 \mathrm{MeV}^{+}$state in ${ }^{28} \mathrm{Si}$ was used. The ratio of the peak areas of the 1.78 and $4.50 \mathrm{MeV}$ transitions is $1.58(3)$, which after applying the $1.0170(15)$ correction for the angular correlation is very close to the value of 1.63(4) from the simulations.

By evaluating Eq. (2) with values from Table I and considering all $325 \mathrm{NaI}$ detector combinations, we obtained $\Gamma_{\gamma}^{E 2} / \Gamma=6.1(6) \times 10^{-4}$.

To reduce dependence on the Monte Carlo evaluation of the absolute efficiencies and perform an analysis similar to that of Obst and Braithwaite [33], the $\Gamma_{\gamma}^{E 2} / \Gamma$ ratio was deduced using

$$
\frac{\Gamma_{\gamma}^{E 2}}{\Gamma}=\frac{N_{020}^{7.65}}{N_{020}^{4.98}} \times \frac{N_{\text {singles }}^{4.98}}{N_{\text {singles }}^{7.65}} \times \frac{\epsilon_{\gamma}^{1.78}}{\epsilon_{\gamma}^{4.44}} \times \frac{\epsilon_{\gamma}^{3.20}}{\epsilon_{\gamma}^{3.21}} \times \frac{W_{020}^{4.98}}{W_{020}^{7.65}} .
$$

The symbols are as given for Eq. (1). An alternative

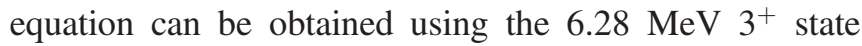
in ${ }^{28} \mathrm{Si}$. Using the singles proton and $p \gamma \gamma$ triple coincidence rates of the 4.98 and $6.28 \mathrm{MeV}$ states, the ratio of the proton to photon efficiencies could be determined. Combining the results from Eq. (3) and using numerical values from Table I, we again obtain $\Gamma_{\gamma}^{E 2} / \Gamma=6.1(6) \times 10^{-4}$.

Using the theoretical total conversion coefficient, $\alpha_{\text {tot }}(E 2,3.21 \mathrm{MeV})=8.77(13) \times 10^{-4}[40]$ and the recommended value of $\Gamma_{\pi}(E 0) / \Gamma \quad$ [25], we obtain $\Gamma_{\mathrm{rad}} / \Gamma=6.2(6) \times 10^{-4}$. This value is more than $3 \sigma$ away from the currently recommended $\Gamma_{\text {rad }} / \Gamma$ value [1]. Most of the previous measurements [29-32] were based on counting the number of ${ }^{12} \mathrm{C}$ atoms surviving after the Hoyle state was formed in various nuclear reactions. To achieve high statistics, the particle detection was carried out without magnetic selection and often with reported count rates above $10 \mathrm{kHz}$. Under these conditions the elimination of accidental coincidences is very challenging.

The investigation by Obst and Braithwaite [33] deduced the $\Gamma_{\gamma}^{E 2} / \Gamma$ ratio using a similar procedure to the present study. Their final result, which was obtained using Eq. (14) of their paper, contains five ratios $(A-E)$. Despite some differences between their experiment and ours, 
TABLE I. Quantities used to evaluate $\Gamma_{\gamma}^{E 2} / \Gamma$ ratio.

\begin{tabular}{lccc}
\hline \hline & $0^{+}(7.65)$ & $0^{+}(4.98)$ & $3^{+}(6.28)$ \\
\hline$N_{\text {020 or 320 }}$ & $217(21)$ & $2233(68)$ & $6295(106)$ \\
$N_{\text {singles }}$ & $2.78(6) \times 10^{8}$ & $1.08(2) \times 10^{6}$ & $3.82(8) \times 10^{6}$ \\
$\gamma$-ray & $\epsilon_{3.21}=0.221(3)$ & $\epsilon_{3.20}=0.222(3)$ & $\epsilon_{4.50}=0.186(3)$ \\
efficiency [\%] & $\epsilon_{4.44}=0.187(3)$ & $\epsilon_{1.78}=0.304(3)$ & $1.0170(15)$ \\
$W_{020 \text { or 320 }}$ & $0.9582(15)$ & $0.9623(15)$ & \\
\hline \hline
\end{tabular}

various combinations of these ratios should agree within a few percent. The largest difference occurs for $B \times D=\left(N_{320}^{6.28} \times N_{\text {singles }}^{4.98}\right) /\left(N_{\text {singles }}^{6.28} \times N_{020}^{4.98}\right) ; \quad$ Ref. [33] reports $0.409(15)$ whereas our value is $0.80(4)$. Thus most of the difference between Obst and Braithwaite [33] and our work stems from the $N_{020}^{4.98} / N_{\text {singles }}^{4.98}$ ratio in the ${ }^{28} \mathrm{Si}$ calibration data. Our results were independently checked in Canberra and Oslo using different analysis software.

Moreover, the data of Obst and Braithwaite for $B \times D$ are not self-consistent. Using the photon efficiencies, the correction factors for the $\gamma \gamma$ angular correlations, and the $\gamma$-ray branching ratio, $B_{\gamma}^{6.28}$ from the $3^{+}$state, we have

$$
B \times D \times \frac{\epsilon_{3.20}}{\epsilon_{4.50}} \times \frac{W_{020}^{4.98}}{W_{320}^{6.28}}=B_{\gamma}^{6.28}=0.882(4) .
$$

The data of Ref. [33] are in disagreement with Eq. (4) by a factor of 2; the present data (Table I) agree within 2\%.

Finally, using the recommended $\Gamma_{\pi}(E 0) / \Gamma$ [25], the adopted $\Gamma_{\pi}^{E 0}$, and our $\Gamma_{\text {rad }} / \Gamma$ values, the radiative width of the Hoyle state is $\Gamma_{\text {rad }}=5.1(6) \times 10^{-3} \mathrm{eV}$. This result suggests a significantly higher radiative width than currently adopted.

The triple-alpha reaction together with ${ }^{12} \mathrm{C}(\alpha, \gamma)$ are the two most important helium burning nuclear reactions with a significant impact on nucleosynthesis and the evolution of massive stars [41-43]. In the core-He burning cycle these reactions compete to determine the relative carbon and oxygen abundances before the core- $\mathrm{C}$ burning starts. The uncertainties due to production rates grow at every step. This makes the uncertainty of the triple-alpha and the ${ }^{12} \mathrm{C}(\alpha, \gamma)$ reaction rates crucial for the production of heavy elements. Recent calculations [42,43] have explored variations within the uncertainties of the production rates: $\pm 10 \%$ for the triple-alpha and $\pm 25 \%$ for the ${ }^{12} \mathrm{C}(\alpha, \gamma)$ reactions. West et al. [42] pointed out that a $25 \%$ increase in the triple-alpha rate would be consistent with a 33\% larger ${ }^{12} \mathrm{C}(\alpha, \gamma)$ rate. Here we report a $34 \%$ change in the triplealpha reaction rate, which is outside of the parameter space of the calculations. This scenario needs to be explored, as it could change many of the model predictions.

In summary, a new measurement of the $\Gamma_{\text {rad }} / \Gamma$ ratio of the Hoyle state has been performed using a much improved experimental setup than used in the last study, more than 40 years ago, giving a value that is significantly higher.
The accurate determination of the triple-alpha rate remains a challenge for low energy nuclear physics. The present experiment only focused on one of the three terms defined in Eq. (1). Confirmation of the new result, using higher resolution photon spectrometers, is well warranted. Additional experiments of the $\Gamma_{\pi}(E 0) / \Gamma$ ratio as well as of the E0 width, $\Gamma_{\pi}(E 0)$ are equally important.

The project was supported by the Australian Research Council Discovery Grants No. DP140102986 and No. DP170101673 and by the Research Council of Norway, Grant No. 263030. T. K., B. A., and A.E. S. acknowledge the hospitality of the University Oslo during the experiments. A. C. L. gratefully acknowledges funding from ERC-STG-2014 under Grant Agreement No. 637686, and support from the "ChETEC" COST Action (CA16117), supported by COST (European Cooperation in Science and Technology).

*Tibor.Kibedi@anu.edu.au

†Present address: Department of Physics, Faculty of Science, King Khalid University, Abha, Saudi Arabia.

"Present address: GSI Helmholtzzentrum für Schwerionenforschung, Darmstadt, Germany, and Helmholtz-Institut Mainz, Mainz, Germany.

${ }^{\S}$ Present address: IFIC, CSIC-Universitat de Valéncia, Valéncia, Spain.

"Present address: Institute of Nuclear Research, MTA ATOMKI, Debrecen, Hungary.

[1] M. Freer and H. O. U. Fynbo, Prog. Part. Nucl. Phys. 78, 1 (2014).

[2] R. Smith, M. Gai, M. W. Ahmed, M. Freer, H. O. U. Fynbo, D. Schweitzer, and S. R. Stern, Phys. Rev. C 101, 021302 (R) (2020).

[3] Y. Funaki, Phys. Rev. C 94, 024344 (2016).

[4] F. Hoyle, Astrophys. J. Suppl. Ser. 1, 12 (1953), http:// adsabs.harvard.edu/full/1954ApJS...1..121H.

[5] D. N. F. Dunbar, R. E. Pixley, W. A. Wenzel, and W. Whaling, Phys. Rev. 92, 649 (1953).

[6] Y. Funaki, Phys. Rev. C 92, 021302(R) (2015).

[7] W. R. Zimmerman et al., Phys. Rev. Lett. 110, 152502 (2013).

[8] R. Garg et al., J. Phys. Conf. Ser. 940, 012043 (2018).

[9] K. D. Launey, A. Mercenne, G. H. Sargsyan, H. Shows, R. B. Baker, M. E. Miora, T. Dytrych, and J. P. Draayer, AIP Conf. Proc. 2038, 020004 (2018). 
[10] C. E. Rolfs and W. S. Rodney, Cauldrons in the Cosmos: Nuclear Astrophysics (University of Chicago Press, Chicago, 1988).

[11] T. Kibédi, A. E. Stuchbery, G. D. Dracoilis, A. Devlin, A. Teh, and K. Robertson, AIP Conf. Proc. 1109, 66 (2009).

[12] J. H. Fregeau, Phys. Rev. 104, 225 (1956).

[13] H. L. Crannell and T. A. Griffy, Phys. Rev. 136, B1580 (1964).

[14] F. Gudden and P. Strehl, Z. Phys. 185, 111 (1965).

[15] H. Crannell, T. A. Griffy, L. R. Suelzle, and M. R. Yearian, Nucl. Phys. A90, 152 (1967).

[16] P. Strehl and Th. H. Schucan, Phys. Lett. 27B, 641 (1968).

[17] P. Strehl, Z. Phys. 234, 416 (1970).

[18] H. Crannell, X. Jiang, J. T. O'Brien, D. I. Sober, and E. Offermann, Nucl. Phys. A758, 399 (2005).

[19] M. Chernykh, H. Feldmeier, T. Neff, P. von NeumannCosel, and A. Richter, Phys. Rev. Lett. 105, 022501 (2010).

[20] F. Ajzenberg-Selove and P. H. Stelson, Phys. Rev. 120, 500 (1960).

[21] D. E. Alburger, Phys. Rev. 118, 235 (1960).

[22] A. W. Obst, T. B. Grandy, and J. L. Weil, Phys. Rev. C 5, 738 (1972).

[23] D. E. Alburger, Phys. Rev. C 16, 2394 (1977).

[24] R. G. H. Robertson, R. A. Warner, and S. M. Austin, Phys. Rev. C 15, 1072 (1977).

[25] T. K. Eriksen, T. Kibédi, M. W. Reed, A. E. Stuchbery, K. J. Cook, A. Akber et al., Phys. Rev. C 102, 024320 (2020).

[26] D. E. Alburger, Phys. Rev. 124, 193 (1961).

[27] P. A. Seeger and R. W. Kavanagh, Nucl. Phys. 46, 577 (1963).

[28] I. Hall and N. W. Tanner, Nucl. Phys. 53, 673 (1964).

[29] D. Chamberlin, D. Bodansky, W. W. Jacobs, and D. L. Oberg, Phys. Rev. C 9, 69 (1974).
[30] C. N. Davids, R. C. Pardo, and A. W. Obst, Phys. Rev. C 11, 2063 (1975).

[31] H.-B. Mak, H. C. Evans, G. T. Ewan, A. B. McDonald, and T. K. Alexander, Phys. Rev. C 12, 1158 (1975).

[32] R. G. Markham, S. M. Austin, and M. A. M. Shahabuddin, Nucl. Phys. A270, 489 (1976).

[33] A. W. Obst and W. J. Braithwaite, Phys. Rev. C 13, 2033 (1976).

[34] C. N. Davids and T. I. Bonner, Astrophys J. 166, 405 (1971), http://adsabs.harvard.edu/full/1971ApJ...166..405D.

[35] M. S. Basunia, Nucl. Data Sheets 114, 1189 (2013).

[36] M. Guttormsen, A. Bürger, T. E. Hansen, and N. Lietaer, Nucl. Instrum. Methods Phys. Res., Sect. A 648, 168 (2011).

[37] M. Guttormsen, A. Atac, G. Løvhøiden, S. Messelt, T. Ramsøy, J. Rekstad, T. F. Thorsteinsen, T. S. Tveter, and Z. Zelazny, Phys. Scr. T32, 54 (1990).

[38] F. Salvat, J. M. Fernndez-Varea, and J. Sempau, PENELOPE2011: A code system for Monte Carlo simulation of electron and photon transport, NEA 6416, 2011, http:// www.oecd-nea.org/upload/docs/application/pdf/2019-12/ nea6416-penelope.pdf.

[39] M. E. Rose, Phys. Rev. 91, 610 (1953).

[40] T. Kibédi, T. W. Burrows, M. B. Trzhaskovskaya, P. M. Davidson, and C. J. Nestor, Nucl. Instrum. Methods Phys. Res., Sect. A 589, 202 (2008).

[41] C. Tur, A. Hager, and S. M. Austin, Astrophys. J. 671, 821 (2007).

[42] C. West, A. Heger, and S. M. Austin, Astrophys. J. 769, 2 (2012).

[43] C. F. Fields, F. X. Timmes, R. Farmer, I. Petermann, W. M. Wolf, and S. M. Couch, Astrophys. J. Suppl. 234, 19 (2018). 